\title{
TRICRITICAL STRUCTURE IN THE ADJOINT HIGGS MODEL?
}

\author{
R. BAIER \\ Fakultät für Physik, Universität Bielefeld, D-4800 Bielefeld, Fed. Rep. Germany \\ R.V. GAVAI ${ }^{1}$ \\ Physics Department, Brookhaven National Laboratory, Upton, NY 11973, USA
}

and

C.B. LANG $^{2}$

Institut für Theoretische Physik, Universität Graz, A-80I0 Graz, Austria

Received 25 February 1986

The SU(2) gauge system with fixed-length Higgs fields is studied in the adjoint representation. High-statistics Monte Carlo simulations on lattice sizes between $L=4$ and $L=8$ give evidence that the transition for large Higgs coupling is of first order. For small Higgs coupling the transition signal may be interpreted as a very weak first- or a second-order transition. In the latter case a hitherto unknown tricritical point in the phase diagram is expected.

Recently interest has focussed on the non-perturbative analysis of gauge-Higgs systems for non-abelian and abelian gauge groups. The lattice formulation of these theories, with the Monte Carlo evaluation of the functional integrals [1] allows to study symmetry breaking and bound-state formation in a very explicit way [2]. Higgs fields are scalars and are implemented on the lattice straightforwardly, in contrast to the elusive fermions. Spontaneous symmetry breaking plays a crucial role in electroweak theories, where the scalars are in the fundamental representation of the gauge group, and also in grand unified theories which often employ scalars in the adjoint representation [3]. Although perturbation theory is found adequate for

1 Supported by the US Department of Energy under Contract No. DE-AC02-76CH00016.

2 Supported by the Florida State University Supercomputer Computations Research Institute which is partially funded by the US Department of Energy through Contract No. DEFCO5-85ER250000; supported in part by Fond zur Förderung der Wissenschaftliche Forschung in Österreich, project P5125. these applications, it is clearly desirable to understand the non-perturbative aspects of these systems. In these studies one may find some interesting fixed-point structure which could lead to a reduction of the input parameters in a dynamical way.

The adjoint Higgs model for the SU(2) gauge group interpolates between the SU(2) pure gauge model and the U(1) gauge model. Studies of the various limiting cases for the fixed Higgs length model show two separate phases: a confinement phase and a phase with broken symmetry where a massless particle lives $[1,4-10]$. The lattice action may be chosen as

$s=S_{\mathrm{G}}+S_{\mathrm{H}}$,

where $S_{\mathrm{G}}$ is the Wilson action [1],

$S_{\mathrm{G}}=-\beta_{\mathrm{F}} \sum_{x, \mu>\nu} \frac{1}{2} \operatorname{Tr}\left(U_{x, \mu} U_{x+\mu, \nu} U_{x+\nu, \mu}^{+} U_{x, \nu}^{+}\right)$,

and

$S_{\mathrm{H}}=-\beta_{\mathrm{H}} \sum_{x, \mu} \frac{1}{2} \operatorname{Tr}\left(\phi_{x} U_{x, \mu} \phi_{x+\mu} U_{x, \mu}^{+}\right)$. 
$U_{x, \mu}$ denotes the link variable associated with the gauge field and it is represented by a $2 \times 2 \mathrm{SU}(2)$ matrix. The adjoint Higgs field $\phi_{x}$ is related to the real triplet field $\phi_{x}, \phi_{x}^{2}=1$, by

$\phi_{x}=\boldsymbol{\sigma} \cdot \boldsymbol{\phi}_{x}$,

where the $\sigma$ are the Pauli matrices. This model can be considered as the $\lambda \rightarrow \infty$ limit of the Higgs model with dynamical length recently investigated $[9,10]$, with the Higgs action given by

$$
\begin{aligned}
S_{\mathrm{H}} & =-\beta_{\mathrm{H}} \sum_{x, \mu} \frac{1}{2} \operatorname{Tr}\left(\phi_{x} U_{x, \mu} \phi_{x+\mu} U_{x, \mu}^{+}-\phi_{x}^{2}\right) \\
& +\lambda \sum_{x}\left(\frac{1}{2} \operatorname{Tr} \phi_{x}^{2}-1\right)^{2} .
\end{aligned}
$$

This form resembles the continuum form more closely.

The fixed length model has been studied with Monte Carlo methods in refs. $[5,6]^{\neq 1}$ on lattices of size $4^{4}$ and $5^{4}$. The resulting phase diagram confirmed the earlier expectations [4]. The $\beta_{\mathrm{H}} \rightarrow \infty$ limit corresponds to a pure $\mathrm{U}(1)$ gauge model, whereas the $\beta_{\mathrm{F}} \rightarrow \infty$ limit reduces to the $\mathrm{O}(3)$ Heisenberg spin model. For $\beta_{\mathrm{F}}=0$ the system is analytically solvable and the case $\beta_{\mathrm{H}}=0$ leaves one with the pure $\mathrm{SU}(2)$ gauge system. The second-order phase transition of the spin model is expected to be connected with the $U(1)$ transition by a phase boundary. In the early analyses $[5,6]$ where this was found to be the case the whole line was conjectured to be second order.

However, recent careful investigations of the U(1) gauge theory $[11,12]$ showed that the $\mathrm{U}(1)$ phase transition for the standard Wilson action is actually of first order. In the plane of fundamental and adjoint (charge-two) representations of the gauge field (with couplings $\widetilde{\beta}_{\mathrm{F}}$ and $\widetilde{\beta}_{\mathrm{A}}$, respectively) this phase transition lies on a line of such first-order phase transitions, presumably ending in a tricritical point $\left(\widetilde{\beta}_{\mathrm{F}}, \widetilde{\beta}_{\mathrm{A}}\right)=$ $(1.09 \pm 0.04,-0.11 \pm 0.05)$ below the Wilson line.

In view of these results it is of interest to study how this first-order phase transition is realized in the discussed gauge Higgs system. It is obvious that somewhere along the line of phase transition that connects the $O(3)$ spin model transition with the $U(1)$ gauge

$\neq 1$ Because of our definition for $S_{H}$ (eq. (3)) our values for $\beta_{\mathrm{H}}$ differ by a factor $1 / 2$ from those of ref. [6]; however we agree with the convention used in ref. [9].
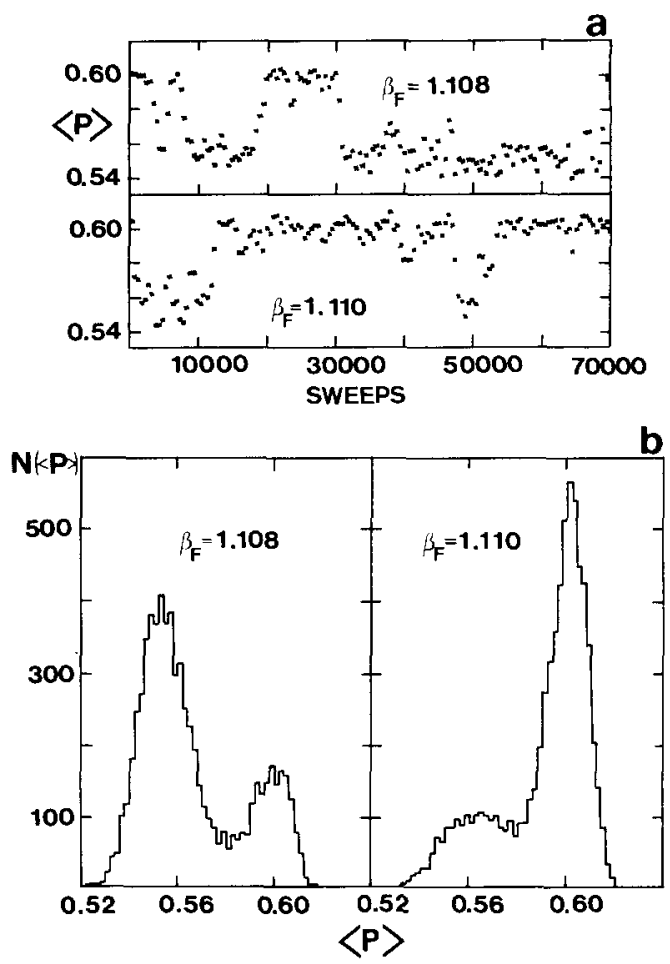

Fig. 1. Time evolution (a) and multiplicity histogram (b) of the plaquette observable $\langle P\rangle$ for lattice size $8^{4}$ at the critical points $\left(\beta_{F}, \beta_{H}\right)=(1.108,10.0)$ and $(1.110,10.0)$, respectively In (a) 500 subsequent configurations are averaged per point, in (b) the values correspond to groups of 10 configurations. The plots represent 70000 configurations.

system the transition changes its type from second to first order. This transition may be realized at $\beta_{\mathrm{F}} \rightarrow \infty$, but if it occurs for a finite value of $\beta_{F}$ one would expect the existence of a so-far unknown tricritical point in the $\beta_{\mathrm{F}}-\beta_{\mathrm{H}}$ plane with an additional relevant parameter. This would imply the existence of another class of fixed points (those lying on the tricritical manifold) with the possibility for a non-trivial continuum limit in addition to the $\beta_{\mathrm{F}} \rightarrow \infty$ limit.

Allowing for further interaction terms in the action (1) like e.g. the adjoint gauge term,

$$
S_{\mathrm{A}}=-\frac{\beta_{\mathrm{A}}}{3} \sum_{x, \mu>\nu} \operatorname{Tr}\left(U_{x, \mu} U_{x+\mu, \nu} U_{x+\nu, \mu}^{+} U_{x, \nu}^{+}\right)^{2} \text {, }
$$

may shed some more light on the situation. A tricritical point in the $\left(\beta_{\mathrm{F}}, \beta_{\mathrm{A}}\right)$ plane of the $\mathrm{U}(1)$ gauge theory (i.e. $\beta_{\mathrm{H}} \rightarrow \infty$ ) [12] implies for our Higgs model that at 
that value of $\beta_{\mathrm{A}}$ the first-order part of the critical line has to disappear. Bhanot and Creitz [13] studied the extended SU(2) model in the $\left(\beta_{F}, \beta_{A}\right)$ plane (i.e. the $\beta_{\mathrm{H}}=0$ limit) and they found a line of phase transitions for $\beta_{\mathrm{A}}$ sufficiently large, pointing towards the $\beta_{\mathrm{F}}$ axis; this line ends at $\left(\beta_{\mathrm{F}}, \beta_{\mathrm{A}}\right)=(1.48 \pm 0.05$, $0.9 \pm 0.03$ ). One may now speculate that this endpoint is connected to the tricritical point for $\beta_{\mathrm{H}} \rightarrow \infty$ by a curve in the $\left(\beta_{\mathrm{F}}, \beta_{\mathrm{A}}, \beta_{\mathrm{H}}\right)$ space which intersects the $\left(\beta_{\mathrm{F}}, \beta_{\mathrm{H}}\right)$ plane. This would lead to a first-order-like behaviour near $\beta_{H}$ and a second-order-like behaviour near large $\beta_{F}$ of the model under discussion.

As a first step towards the understanding of this structure we study in more detail the $\beta_{\mathrm{A}}=0$ plane; i.e. the gauge-Higgs model for fixed length Higgs fields, as given in eqs. (1)-(3). We perform Monte Carlo calculations for various values of $\left(\beta_{\mathrm{F}}, \beta_{\mathrm{H}}\right)$ on periodic lattices of size $L^{4}$, with $L$ between $L=4$ and $L=8$. The full group $\mathrm{SU}(2)$ has been employed, but for computational convenience we fix the gauge for the Higgs field (4) by $\boldsymbol{\phi}_{x}=(0,0,1)$. First the critical line is investigated by simulations on $L=4,5$ and 6 lattices, using both ordered and disordered starts. The runs contain 10000-12000 $(L=4,5)$, and $20000(L=6)$ iterations, respectively. We find that our estimates of the critical couplings $\left(\beta_{\mathrm{F}}, \beta_{\mathrm{H}}\right)$ are in agreement with the results of refs. $[5,6]$. For a set of representative critical couplings we explore the $L=8$ lattice with high statistics in the range of 70000 or more iterations. We measure the Wilson action (2) per plaquette,

$\langle P\rangle=\left\langle\frac{1}{2} \operatorname{Tr} U U U^{+} U^{+}\right\rangle$,

as well as the gauge-Higgs interaction (3) per link,

$\langle L\rangle=\left\langle\frac{1}{2} \operatorname{Tr} \phi U \phi U^{+}\right\rangle$.

Their multiplicity distributions are determined by averaging over ten successive iterations.

Following the discussion given above the results are arranged in two groups: those on the branch $\beta_{H}>1.0$ approaching the $U(1)$ model for $\beta_{H} \rightarrow \infty$, and those for $\beta_{\mathrm{F}}>2.2$ leading towards the Heisenberg spin model for finite $\beta_{\mathrm{F}}$.

Let us first discuss our results on the branch connected to the $U(1)$ transition for a large value of $\beta_{H}$, namely $\beta_{\mathrm{H}}=10.0$. Fig. 1 shows the time development and multiplicity histogram of the plaquette values $\langle P\rangle$ for lattice size $8^{4}$ obtained for two values of the critical coupling, $\beta_{\mathrm{F}}=1.108$ and $\beta_{\mathrm{F}}=1.110$, respectively. Con-

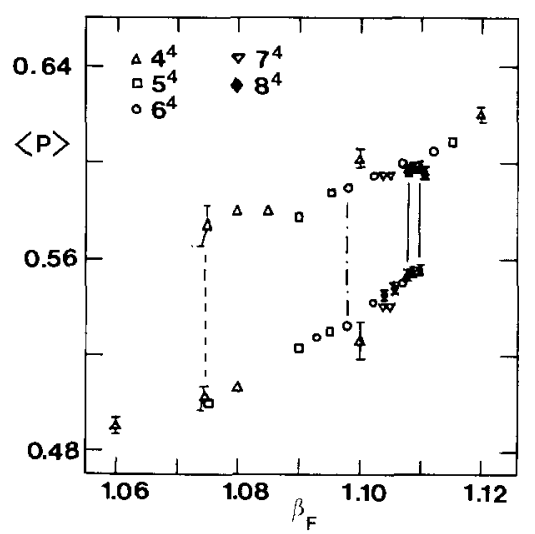

Fig. 2. The plaquette values $\langle P\rangle$ as a function of $\beta_{F}$ determined for various lattice sizes on the upward branch of the critical line at $\beta_{\mathbf{H}}=10.0$.

sidering the 70000 subsequent configurations (fig. 1a) there is a clear evidence of metastability. Tunnelings occur between two states, which are reflected in the double-peak structure in the histogram $N(\langle P\rangle)$ presented in fig. $1 \mathrm{~b}$. The tunneling frequency depends on the size of the lattice, e.g. the flip between the states occurs rather often, sometimes every 1000-2000th configuration on the smaller lattices $4^{4}-6^{4}$, which is to be compared with the behaviour on $8^{4}$ given in fig. $1 \mathrm{a}$.

Fig. 2 summarizes the obtained averaged plaquette values $\langle P\rangle$ for various lattice sizes. One sees a hysteresis whose width decreases as the volume of the system increases: on $4^{4} \beta_{F} \simeq 1.075 \rightarrow 1.110$, on $6^{4} \beta_{F} \simeq$ $1098 \rightarrow 1.110$, whereas on $8^{4}$ the width becomes as small as $\beta_{\mathrm{F}} \simeq 1.108 \rightarrow 1.110$. The gap in $\langle P\rangle$ decreases slightly with increasing $L$, however, its size dependence is not strong enough to expect a complete vanishing for infinite size. On the $8^{4}$ lattice we estimate a gap. $\Delta\langle P\rangle \simeq 0.043$ for $\beta_{\mathrm{F}}=1.108 \rightarrow 1.110$ (figs. $1 \mathrm{~b}$ and 2 ). Thus these results indicate a first-order transition at $\beta_{H}=10.0$, which was not seen in the previous works $[5,6]$. However, our claim is in accord with the transition in the $U(1)$ gauge system $[11,12]$, which is the limiting model for $\beta_{\mathrm{H}} \rightarrow \infty$.

On the $6^{4}$ lattice we follow the critical line towards smaller values of $\beta_{\mathrm{H}}$. We find indications of metastability in the time development of $\langle P\rangle$ and in its multiplicity distribution $N(\langle P\rangle)$ at the following couplings: $\left(\beta_{\mathrm{F}}, \beta_{\mathrm{H}}\right)=(1.1375,7.5),(1.210,5.0),(1.2825,3.75)$ 
and $(1.4825-1.4875,2.25)$. Concerning the gap $\Delta\langle P\rangle$ no significant decrease down to $\beta_{\mathrm{H}}=2.25$ is observed. This result is in agreement with that of ref. [9], in which it is concluded that the phase transition is weakly first order at $\beta_{F}=1.5$ for a wide range of $\lambda$-couplings, including the fixed length case $\lambda \rightarrow \infty$ : the plaquette data obtained on a $6^{4}$ lattice with even higher statistics than ours show a jump in $\langle P\rangle$, with $\Delta\langle P\rangle \simeq 0.05$, at $\beta_{\mathrm{F}}=$ 1.5, $\beta_{\mathrm{H}} \simeq 2.2$ and $\lambda \rightarrow \infty$ (fig. 7 of ref. [9]).

When decreasing $\beta_{\mathrm{H}}$ down to $\beta_{\mathrm{H}}=1.0$ hysteresis cycle runs indicate the transition to occur between $2.575 \leqslant \beta_{\mathrm{F}} \leqslant 2.625$ on the $6^{4}$ lattice. However, a closer examination of the behaviour of $\langle P\rangle$ and $\langle L\rangle$ does no more reveal any sign of metastability. Consequently the gap $\Delta\langle P\rangle$ seems to vanish in the range $2.25>\beta_{\mathrm{H}}>1.0$ according to our presently available statistics for the $6^{4}$ lattice.

Let us turn to the part of the transition line that ends at the pure $O(3)$ model transition at $\beta_{F} \rightarrow \infty$. We concentrate on one value of $\beta_{\mathrm{F}}=3.0$. The phase transition occurs now in the direction $\beta_{\mathrm{H}}$ and it is most clearly observed in the corresponding observable: the link variable $\langle L\rangle$. However, the signal itself is far from being completely clear (cf. related remarks by Karsch et al. [7]).

In fig. 3 we exhibit the time evolution and the corresponding histogram for $\langle L\rangle$ obtained at $\beta_{\mathrm{F}}=3.0$ and the presumed value of $\beta_{\mathrm{H} \text {,crit }}=0.6355$ for 160000 subsequent configurations on the $8^{4}$ lattice. There is no indication of two overlapping states (also in the corresponding distributions for $\langle P\rangle)$. The same holds true for neighbouring values $\beta_{\mathrm{H}}=0.63475$ and $\beta_{\mathrm{H}}=$ 0.63575 , respectively. There are also no two-state signals seen on the smaller lattices in the vicinity of the critical couplings: $\beta_{\mathrm{F}}=0.67-0.71$ for $L=4, \beta_{\mathrm{F}}=$ $0.65-0.67$ for $L=5$ and $\beta_{\mathrm{F}} \simeq 0.6525$ for $L=6$, although the statistics is less than for the $8^{4}$ lattice. We therefore conclude that for the range of lattice sizes investigated (up to $8^{4}$ ) there is no signal of a firstorder transition. However, we cannot exclude that such a signal may evolve on substantially larger lattices (cf. ref. [14]). Even if that happens the latent heat would be very small: we estimate on $8^{4}$ a gap $\Delta\langle L\rangle$ in the link observable to be less than 0.004 . Our statistical errors on $\langle L\rangle$ are presently still too large, e.g. at $\beta_{\mathrm{H}}=0.6355$ (fig. 3) we obtain $\langle L\rangle=0.2667 \pm$ 0.0011 leaving out the first 20000 iterations for equilibration, in order to resolve uniquely a possible coexistance of metastable states at $\beta_{F}=3.0$.
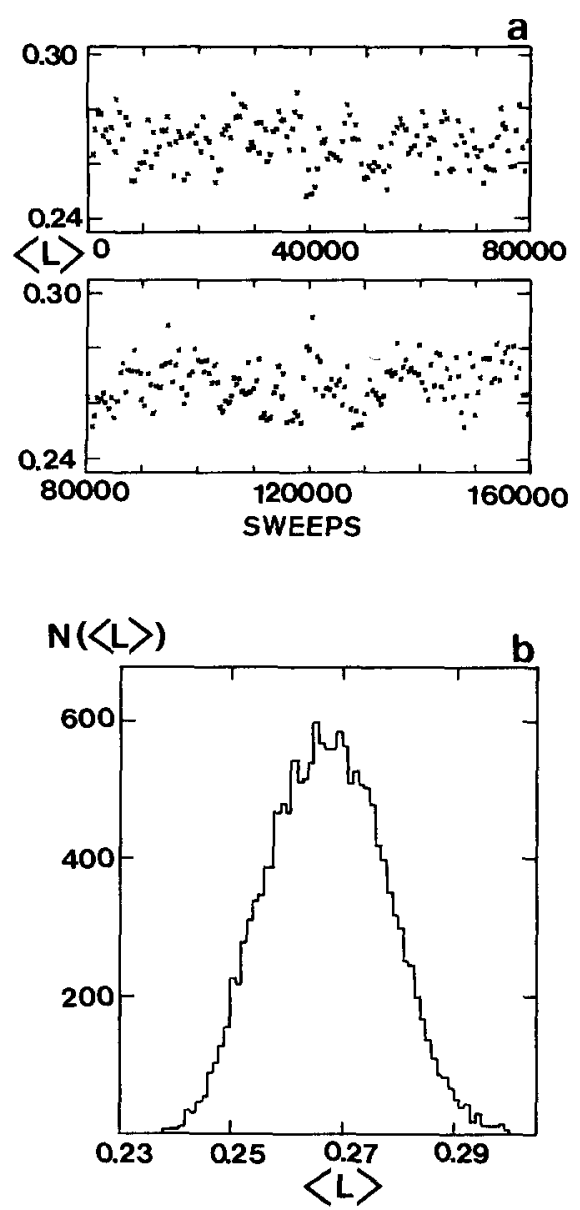

Fig. 3. Time evolution (a) and multiplicity histogram (b) of the link observable $\langle L\rangle$ for lattice size $8^{4}$ at the critical point $\left(\beta_{F}, \beta_{H}\right)=(3.0,0.6355)$. The sampling is performed as described in fig. 1; the plots here correspond to 160000 configurations.

For the moment we think it more probable that this branch (for $\beta_{\mathrm{F}}>2.2$ ) of the phase transition is actually second order, although attempts to strengthen this statement by investigating finite-size scaling turn out to be not very conclusive on the basis of the accumulated statistics. The critical couplings $\beta_{H}$ given above for different lattice sizes are compatible with $\beta_{\mathrm{Hcrit}}^{\mathrm{L}}-\beta_{\mathrm{Hcrit}}^{\infty} \propto \mathrm{L}^{-1 / \nu}$, with $\nu=1 / 2-$ the critical index of the $O(3)$ spin model. However, the maximum height of the link contribution to the normalized specific heat (analogously defined as the one for the plaquette contribution given in ref. [15]) is very slowly increasing for increasing lattice size: for $6^{4}$ to $8^{4}$ it is 
even compatible with being constant, although an increase proportional to $\ln L$ (for $\nu=1 / 2$ ) is to be expected. All this might be an indication of yet insufficient statistics and too small lattices for performing a reliable finite size scaling analysis.

We have performed a high-statistics study for the SU(2) gauge-Higgs model with Higgs fields of fixed length in the adjoint representation of SU(2). We find a clear evidence for a first-order phase transition for that part of the critical line that joins the $U(1)$ gauge system transition at $\beta_{H}=\infty$. On the other part of the transition line the results are compatible with a secondorder transition signal, although presently we are not able to prove it. Nevertheless, this model is obviously a possible candidate for a lattice field theory model with tricritical structure. Further studies are necessary and should be performed on larger lattices, $L>8$, and with renormalization group methods in order to identify or rule out the existance of a tricritical point in the region $1.5<\beta_{\mathrm{F}}<2.5$ and $1.0<\beta_{\mathrm{H}}<2.25$ of the phase diagram in the $\beta_{\mathrm{F}}-\beta_{\mathrm{H}}$ plane.

C.B.L. wants to thank the Supercomputer Computations Research Institute (SCRI) of Florida State University, Tallahassee, for the kind hospitality granted in summer 1985. The calculations have been performed at the Rechenzentrum der Ruhr-Universität Bochum (Cyber 205), at Universität Köln (Cyber 176), at Brookhaven National Laboratory (Cyber 7600) and at SCRI, Florida State University Tallahassee (Cyber 205). We want to thank all those institutions and their technical staff for the support.
References

[1] K.G. Wilson, Phys. Rev. D10 (1974) 2445; M. Creutz, L. Jacobs and C. Rebbi, Phys. Rep. 95 (1983) 201;

J.M. Drouffe and J.-B. Zuber, Phys. Rep. 102 (1983) 1; C. Rebbi, Lattice gauge theories and Monte Carlo simulations (World Scientific, Singapore, 1983).

[2] J. Jersák, Review talk Workshop on Lattice gauge theories (Wuppertal, FRG, November 1985), preprint PITHA $85 / 25$.

[3] H. Georgi and S.L. Glashow, Phys. Rev. Lett. 28 (1972) 1494.

[4] K. Osterwalder and E. Seiler, Ann. Phys. 110 (1978) 440;

E. Fradkin and S. Shenker, Phys. Rev. D19 (1979) 3682; E. Seiler, Gauge theories as a problem of constructive quantum field theory and statistical mechanics, Lecture Notes in Physics, Vol. 159 (Springer, Berlin, 1982).

[5] C.B. Lang, C. Rebbi and M. Virasoro, Phys. Lett. B 104 (1981) 294.

[6] R. Brower, D. Kessler, T. Schalk, H. Levine and M. Nauenberg, Phys. Rev. D25 (1982) 3319.

[7] F. Karsch, E. Seiler and I. Stamatescu, Phys. Lett. B 131 (1983) 139.

[8] J.M. Drouffe, J. Jurkiewicz and A. Krzywicki, Phys. Rev. D29 (1984) 2982.

[9] I.-H. Lee and J. Shigemitsu, Nucl. Phys. B 263 (1986) 280.

[10] G. Schierholz, J. Seixas and M. Teper, Phys. Lett. B 151 (1985) 69.

[11] J. Jersák, T. Neuhaus and P.M. Zerwas, Phys. Lett. B 133 (1983) 103.

[12] H.G. Evertz, J. Jersák, T. Neuhaus and P.M. Zerwas, Nucl. Phys. B 251 [FS13] (1985) 279.

[13] G. Bhanot and M. Creutz, Phys. Rev. D24 (1981) 3212.

[14] W. Langguth and I. Montvay, Phys. Lett. B 165 (1985) 135 ;

W. Langguth, I. Montway and P. Weisz, preprint DESY 85-138 (December 1985).

[15] B. Lautrup and M. Nauenberg, Phys. Lett. B 95 (1980) 63. 